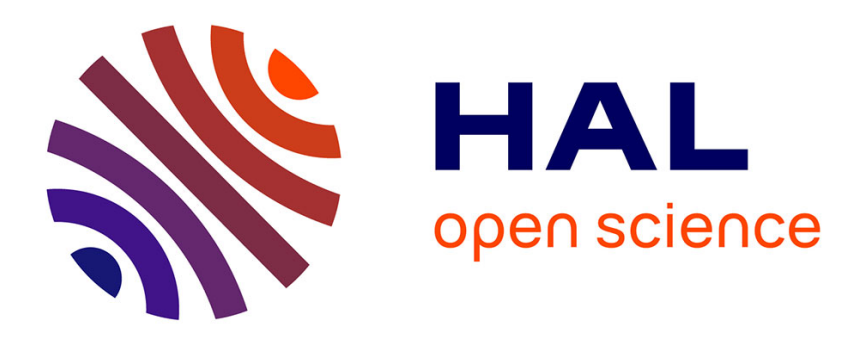

\title{
Soret motion in non-ionic binary molecular mixtures
} Yves Leroyer, Alois Würger

\section{To cite this version:}

Yves Leroyer, Alois Würger. Soret motion in non-ionic binary molecular mixtures. Journal of Chemical Physics, 2011, 135 (5), pp.054102. 10.1063/1.3615954 . hal-00617280

\section{HAL Id: hal-00617280 \\ https://hal.science/hal-00617280}

Submitted on 26 Aug 2011

HAL is a multi-disciplinary open access archive for the deposit and dissemination of scientific research documents, whether they are published or not. The documents may come from teaching and research institutions in France or abroad, or from public or private research centers.
L'archive ouverte pluridisciplinaire HAL, est destinée au dépôt et à la diffusion de documents scientifiques de niveau recherche, publiés ou non, émanant des établissements d'enseignement et de recherche français ou étrangers, des laboratoires publics ou privés. 


\title{
Soret motion in non-ionic binary molecular mixtures
}

\author{
Yves Leroyer and Alois Würger \\ Laboratoire Ondes et Matière d'Aquitaine, Université Bordeaux 1 \& CNRS, \\ 351 cours de la Libération, 33405 Talence, France
}

\begin{abstract}
We study the Soret coefficient of binary molecular mixtures with dispersion forces. Relying on standard transport theory for liquids, we derive explicit expressions for the thermophoretic mobility and the Soret coefficient. Their sign depends on composition, the size ratio of the two species, and the ratio of Hamaker constants. Our results account for several features observed in experiment, such as a linear varition with the composition; they confirm the general rule that small molecules migrate to the warm, and large ones to the cold.
\end{abstract}

corresponding author: a.wuerger@loma.u-bordeaux1.fr

PACS numbers:

\section{INTRODUCTION}

The Soret effect describes the non-uniform composition of a complex fluid or molecular mixture in a temperature gradient [1]. In the steady state one observes that some components accumulate at the cold side of the sample, whereas others rather migrate in the opposite direction [2-8]. This thermophoresis, or thermal diffusion, is driven by thermodynamic forces that act on the different components.

For charged colloids in an electrolyte solution, several mechanisms have been singled out: The temperature gradient induces a non-uniform pressure and permittivity in the electric double layer, which drive the particle to the cold [9]. On the other hand, the Seebeck effect of the electrolyte solution induces a macroscopic electric field which imposes on a suspended particle an electrophoretic velocity that may take either sign, depending on the electrolyte properties [10]. Standard colloidal transport theory provides an overall satisfactory picture for the thermophoretic velocity, and in particular describes its variation with the salinity and the $\mathrm{pH}$ value $[11,12]$.

Surprisingly, the situation is less clear for mixtures of non-ionic molecules and dilute polymer solutions, although dispersion forces are the only molecular interaction. We mention several aspects that add complexity in the case of a binary mixture as illustrated in Fig. 1. First, molecular mixtures generally comprise comparable volume fractions of their components and thus cannot be treated in the low-dilution limit [13-18]. Second, in charged systems macroscopic hydrodynamics works well, since both the Debye length and the solute size are large compared to the solvent molecular structure [6-8]; this continuum approximation is less obvious for short-ranged dispersion forces [19]. Third, as a related aspect, the interaction energies of each molecular species are comparable; thus it is not suffiicient to consider the forces acting on a "solute" molecule; both components have to be treated on an equal footing, and the Soret motion arises from the competition of their transport velocities $[20,21]$. Finally we note that, contrary to the case of col-

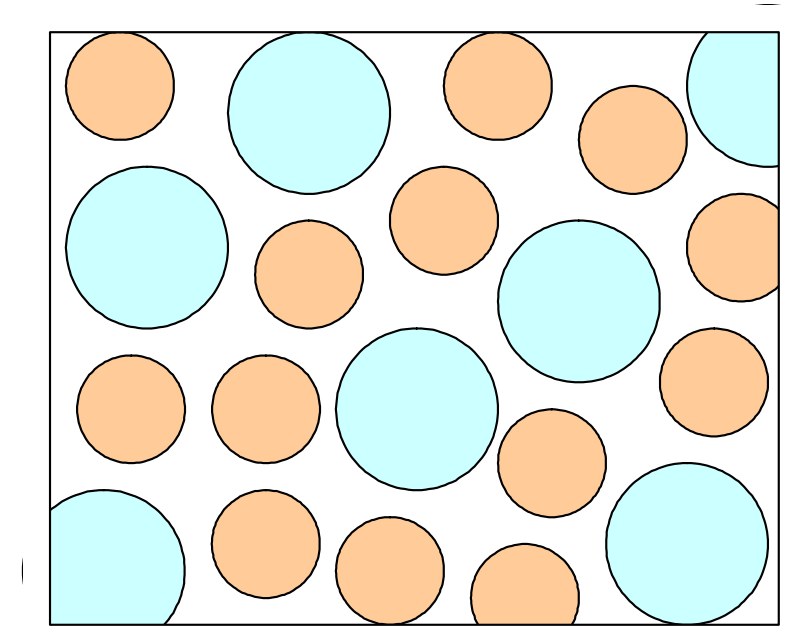

FIG. 1: Schematic view of a binary mixture of spherical beads. In a mean-field approach, the transport coefficients are calculated for each species in a homogeneous liquid.

loidal particles, the kinetic energy is important in molecular mixtures, and contributes significantly to the thermal diffusion [22]. These aspects have been addressed by previous authors, mainly in terms of heat-of-transfer models [23-26] and numerical simulations of the molecular dynamics [27-34]

In this paper we study the Soret mobility in binary mixtures. From the condition of mass conservation, we derive in section 2 a formal expression for the Soret coefficient that depends on the thermal transport and diffusion coefficients $\xi_{i}$ and $D_{i}$ of the two components. Using van der Waals potentials and low-Reynolds number hydrodynamics, we calculate in section 3 the $\xi_{i}$ and in particular evaluate their dependence on the molecular size. In Section 4 we discuss our main results in view of the most important parameters and compare with experimental findings. 


\section{THE SORET COEFFICIENT}

We consider a binary system with volume fractions $\phi_{1}=\phi$ and $\phi_{2}=1-\phi$. For the sake of simplicity we suppose that the mass density does not depend on the composition, in other words, that both components have the same density as the binary mixture. Yet note that the density does depend on temperature.

The diffusion kinetics with respect to composition changes are expressed in terms of the current related to $\phi[1]$,

$$
J_{1}=-D \nabla \phi-\phi(1-\phi) D_{T} \nabla T
$$

where $D$ is the mutual diffusion coefficient of the two components; the drift term is proportional to the temperature gradient with the mobility $D_{T}$. In the case of a closed system in contact with two heat reservoirs at different temperatures, this current vanishes in the stationary state; the spatial variation of the molar fraction is described by the Soret coefficient

$$
S_{T}=\frac{D_{T}}{D} .
$$

If both $S_{T}$ and $\nabla T$ are constant, Eq. (1) can be integrated in closed form. In the dilute limit $\phi \rightarrow 0$, colloidal or macromolecular dispersions are well described by calculating both the Einstein coefficient and the mobility for a single suspended particle from the solute-solvent interactions.

The above Eq. (1) follows from rather general considerations on heat and mass flows in a non-uniform temperature [1], yet does not give explicit expressions for $D$ and $D_{T}$. In order to relate these coefficients to the physical parameters of the liquid, we rewrite the currents of each component as

$$
J_{i}=-D_{i} \nabla \phi_{i}+\phi_{i} u_{i}-\phi_{i} \bar{u},
$$

where $D_{i}$ describes the diffusive motion of a given molecule, $u_{i}$ its drift velocity, and $\bar{u}$ is the center-of-mass velocity $\bar{u}$. In the simplest case the tracer diffusion coefficient reads as

$$
D_{i}=\frac{k_{B} T}{6 \pi \eta R_{i}}
$$

where $\eta$ is the viscosity of the mixture and $R_{i}$ the hydrodynamic radius. The transport velocity accounts for thermally driven motion in a temperature gradient,

$$
u_{i}=-\xi_{i} \nabla T,
$$

with the molecular mobility $\xi_{i}$ of compenent $i$. The center-of-mass or background velocity $\bar{u}$ assures the condition $J_{1}+J_{2}=0$ which results from mass conservation [1]; for thermal diffusion in electrolyte solutions its role has been spelt out by Kirkwood and co-workers [35, 36].

Here we are intereseted in the steady state where the current of each component vanishes separately, $J_{1}=0=$
$J_{2}$. Eliminating the gradients $\nabla \phi_{1}=-\nabla \phi_{2}$ from (3) and solving the remaining equation for the center-of-mass velocity, we find

$$
\bar{u}=\frac{u_{1} \phi_{1} / D_{1}+u_{2} \phi_{2} / D_{2}}{\phi_{1} / D_{1}+\phi_{2} / D_{2}} .
$$

This expression is the average of the quanitities (5), with the weight of each term is given by the volume fraction divided by the diffusion coefficient.

Replugging $\bar{u}$ in (3) one obtains the current of component 1 ,

$$
J_{1}=-\left(\phi_{1} D_{2}+\phi_{2} D_{1}\right) \nabla \phi_{1}+\phi_{1} \phi_{2}\left(u_{1}-u_{2}\right),
$$

and comparing with (1) one readily identifies the quantities $D_{T}$ and $D$. The thermal diffusion coefficient is given by the difference of the mobilities of the two species,

$$
D_{T}=\xi_{1}-\xi_{2}
$$

This implies that the species with the larger mobility moves to the cold, e.g., for $\xi_{2}>\xi_{1}$ we have $D_{T}<0$ for component 1 . It turns instructive to consider the particular case $\xi_{1}=0$. The the thermophoretic mobility $D_{T}$ of component 1 is entirely determined by the transport coefficient $\xi_{2}$. Eq. (7) has a simple physical meaning: In general the molecules of component 2 have a tendency to migrate to the cold $\left(\xi_{2}>0\right)$; as a consequence, they push those of species 1 in the opposite direction towards higher temperature.

The mutual diffusion coefficient arises as the weighted mean of the molecular coefficients,

$$
D=\phi_{1} D_{2}+\phi_{2} D_{1}
$$

This corresponds to the expression of a simple model developped for molecular mixtures $[37,38]$. A more complex result arises when including in the currents (3) additional terms, such as the concentration gradients of other species $D_{i j} \nabla c_{j}$.

Thus we obtain the Soret coefficient

$$
S_{T}=\frac{\xi_{1}-\xi_{2}}{\phi_{1} D_{2}+\phi_{2} D_{1}} .
$$

Except for the sign, this expression is symmetric under exchange of the two species; thus we have $-S_{T}$ for component 2. Eqs. (7)-(9) constitute the main result of the formal part of this paper. In the remainder we calculate the mobility $D_{T}$.

\section{DISPERSION FORCES}

In this section we evaluate the mobility $\xi$ for a single solute molecule interacting with solvent through dispersion forces. The main issue is the variation of $\xi$ with the size of the solute particle. 
The interaction energy between the particle and the surrounding liquid is obtained by integrating the van der Waals potential over their respective volumes,

$$
\varphi=-\frac{H}{\pi^{2}} \int \frac{d V d V^{\prime}}{\left|\mathbf{r}-\mathbf{r}^{\prime}\right|^{6}}
$$

where the Hamaker constant takes values $H \sim 10^{-20} \mathrm{~J}$, which at room temperature corresponds to several $k_{B} T$. This form has been used for calculating the dispersion forces between two macroscopic bodies [39].

For the hydrodynamic treatment we rather need the potential energy density in the liquid phase. In turns out convenient to evaluate the integral in $\varphi$ for the effective volume $V_{S}$ occupied by a single liquid molecule at position $\mathbf{r}$. Replacing this volume with the inverse number density of liquid molecules, $V_{S}=1 / c$, we have

$$
c \varphi(\mathbf{r})=-\frac{H}{\pi^{2}} \int \frac{d V^{\prime}}{\left|\mathbf{r}-\mathbf{r}^{\prime}\right|^{6}},
$$

where the remaining integral runs over the solute particle.

The van der Waals potential acting on each liquid molecule results in an excess pressure $P=-c \varphi$ close to the solid surface. The concentration $c$ of the liquid phase, and thus the pressure $P$, vary along the solute surface, giving rise to motion of the surrounding fluid. In the following we first consider the simple case of a flat solid boundary and then generalize to a spherical solute particle of finite size.

\section{A. Flat interface}

In the case of a large solute, the volume integral in (11) can be taken over an infinite half space. The resulting expression is readily evaluated, and gives the well-known result [39]

$$
c \varphi(z)=-\frac{H}{6 \pi z^{3}},
$$

where $z$ indicates the distance from the interface. Note that because of the thermal gradient, the concentration of solvent molecules $c(x)$ varies along the surface.

The fluid dynamics is governed by Stokes' equation. In the vicinity of a flat surface, it takes a particularly simple form which we discuss in terms of local coordinates $x$ and $z$ as defined in Fig. 2. The vertical velocity component vanishes, whereas a finite velocity $v_{x}$ occurs along the surface and obeys $\eta \partial_{z}^{2} v_{x}=\partial_{x} P$. With Stokes boundary conditions this equation is readily integrated [19, 40], and gives the velocity well beyond the range of interaction of the van der Waals potential,

$$
v_{B}=-\frac{1}{\eta} \frac{d c}{d x} \int_{d_{0}}^{\infty} d z z \varphi(z) .
$$

The lower bound $d_{0}$ is required in order to assure convergence of the integral. In physical terms, this cut-off
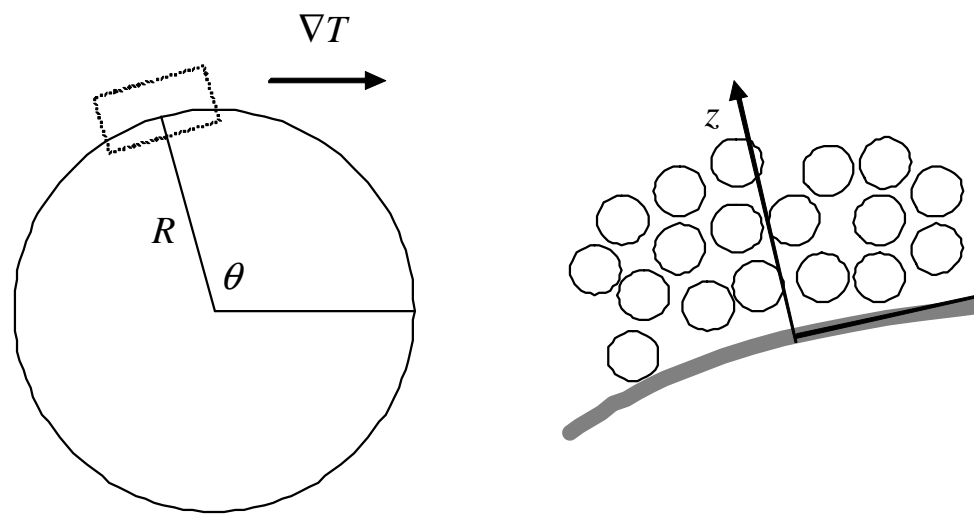

FIG. 2: Boundary layer approximation at the surface of a solute particle.

parameter accounts for the minimal distance of atoms of the liquid and the particle.

Performing the integral in (13) gives $H /\left(6 \pi d_{0}\right)$. In a non-uniform temperature the concentration varies as

$$
\nabla c=-c \beta \nabla T
$$

with the thermal expansion coefficient $\beta$. Thus we obtain the quasislip velocity of the liquid

$$
v_{B}=\frac{H \beta}{6 \pi \eta d_{0}} \frac{d T}{d x} .
$$

In the frame attached to the solid boundary, the liquid flows to higher temperatures.

\section{B. Spherical particle}

Now we account for a finite solute volume in the van der Waals potential (11). In the case of a homogeneous spherical particle of radius $R$, the integral is readily performed,

$$
c \varphi(\mathbf{r})=-\frac{4 H_{1}}{3 \pi} \frac{R^{3}}{\left(r^{2}-R^{2}\right)^{3}} .
$$

In the limit $R \rightarrow \infty$ one readily recovers with $z=r-R$ the expression for a macroscopic body, Eq. (12). Strictly speaking, Stokes' equation no longer separates in parallel and normal components; using (13) close to a curved surface corresponds to the boundary layer approximation [40].

Here we describe finite-curvature corrections that arise from the modified van der Waals potential (16). Inserting the vertical distance $z=r-R$ in (13) and performing the integral one finds

$$
v_{B}=\frac{H \beta}{6 \pi \eta d_{0}} \frac{d T}{d x} F\left(\frac{d_{0}}{R}\right)
$$


(10) is based on a continuum approximation that ignores the molecular structure and thus leads to a divergency

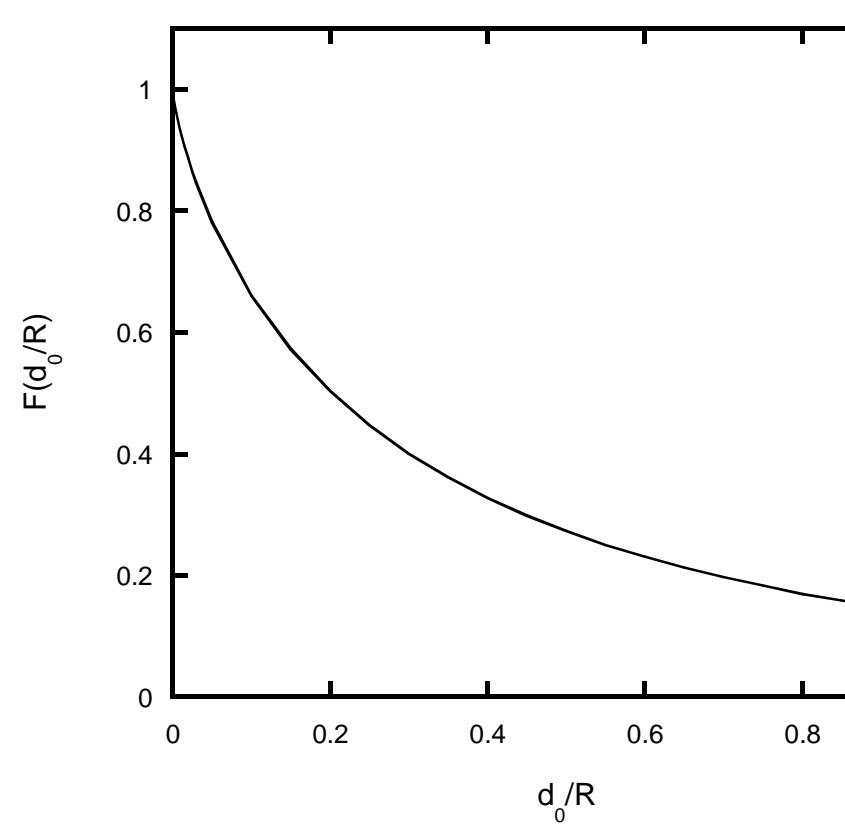

interaction energy if two beads of volumes $V$ and $V^{\prime}$ are in contact. The common practice of regularization consist in a cut-off parameter that accounts for the finite distance of the two bodies; in a physical picture $d_{0}$ may be viewed as the minimum distance of atoms of different beads, and thus is of the order of an Angström.

In Eq1. (13) the length $d_{0}$ takes a somewhat different meaning. The integral gives the solution of the parallel component of Stokes' equation, which describes the balance of viscous and dispersion forces along the particle surface; the integrand is related to the shear stress in the vicinity of the bead under consideration. Thus $d_{0}$ accounts for the length scale where continuum hydrodynamics cease to be valid, and is related to the molecular s.ze; the notion of shear flow or velocity gradient is mear ingless at shorter distances, as illustrated by the right panel of Fig. 2.

In the case of a large solute particle, $R_{1} \gg R_{2}$, it is save to identify $d_{0}$ with the size of a solvent molecule $R_{2}$. Its value is less obvious if both components are of similar size. The length $d_{0}$ subsumes the cut-off of macrosccopic hydrodynamics on a molecular scale. Using the relation $d_{0} \approx R$ in the form factor one finds that $F$ takes a value

FIG. 3: The function $F\left(d_{0} / R\right)$ in the range $0 \leq d_{0} / R \leq 1$. In the limit $x \rightarrow 0$ one recovers with $F(0)=1$ the mobility of large particles. The function $F$ strongly decreases as the particle radius $R$ becomes comparable to the cut-off parameter $d_{0}$.

where the finite size of the bead is accounted for by

$$
F(x)=\frac{3}{2} x \ln \left(\frac{x}{x+2}\right)+\frac{3 x^{2}+9 x+4}{(x+2)^{2}}
$$

with the shorthand notation $x=d_{0} / R$. For large particles $(R \rightarrow \infty)$ this function takes the value $F(0)=1$, and decreases monotonically as the radius $R$ becomes smaller. This is due to the fact that a smaller particle volume exerts a weaker force on the surrounding fluid.

The projection of the constant macroscopic temperature gradient $\nabla T$ on the particle surface varies with the polar angle $\theta$ defined in Fig. 2. The corresponding orientational average of the velocity vector $v_{B}$ results in a factor $\frac{2}{3}$ [40]; noting moreover that the particle moves in the direction opposite to the surrounding fluid, one finds

$$
\xi=\frac{2 H \beta}{9 \pi \eta d_{0}} F\left(\frac{d_{0}}{R}\right) .
$$

In the limit of large particles $\left(R \gg d_{0}\right)$ we recover the result given previously in Ref. [19].

\section{The cut-off parameter $d_{0}$}

The physical meaning of the cut-off parameter introduced in Eq. (13) is twofold. The van der Waals potential of about $15 \%$.

\section{BINARY SYSTEMS}

Here we discuss the above results in view of experimental findinigs on binary molecular mixtures and polymer solutions. The mobilities $\xi_{i}$ are calculated from a mean-field model, where each molecule is considered as a spherical bead that is surrounded by a continuous solvent consisting of molecular species 1 and 2 of volume fractions $\phi_{i}$. Thus the transport coefficients $\xi_{i}$ are given by Eqs. (7) and (19), providing the general expression for the thermophoretic mobility,

$$
D_{T}=\frac{2 \beta}{9 \pi \eta d_{0}}\left[H_{1} F_{1}-H_{2} F_{2}\right]
$$

with the appropriate shape factors $F_{i}=F\left(d_{0} / R_{i}\right)$. In a simple mean-field picture the Hamaker constants of solute and solvent beads read as

$$
\begin{aligned}
& H_{1}=\phi_{1} H_{11}+\phi_{2} H_{12}, \\
& H_{2}=\phi_{1} H_{12}+\phi_{2} H_{22},
\end{aligned}
$$

where $H_{i j}$ describes the dispersion forces between materiels $i$ and $j$.

Experiments on molecular mixtures give the Soret coefficient rather than the mobility $D_{T}$. Inserting the StokesEinstein relation $D_{i}=k_{B} T / 6 \pi \eta R_{i}$ in the mutual diffusion coefficient one finds

$$
S_{T}=\beta \frac{4 R_{\mathrm{eff}}}{3 d_{0}} \frac{H_{1} F_{1}-H_{2} F_{2}}{k_{B} T},
$$


where we have introduced the shorthand notation for the effective radius

$$
\frac{1}{R_{\mathrm{eff}}}=\frac{\phi_{1}}{R_{2}}+\frac{\phi_{2}}{R_{1}}
$$

Both $R_{\text {eff }}$ and the Hamaker constants depend on the volume fractions, thus giving rise to an intricate variation with the composition. The mobility $D_{T}$ and the Soret coefficient $S_{T}$ may take either sign, depending on the relative values of the $H_{i j}$ and $F_{i}$.

\section{A. Large particles}

We start with the case of a dilute suspension of large solute particles $P$ in a molecular solvent $S$, as illustrated in Fig. 2. With $R_{P} \gg d_{0}$ and $\phi_{P} \ll 1$ we find that the thermophoretic mobility

$$
D_{T}=\frac{2 \beta}{9 \pi \eta d_{0}}\left(H_{P S}-F_{S} H_{S S}\right) .
$$

is independent of the solute size $R_{P}$. This is a particular case of a generally valid statement, which has been shown first for electrophoresis of particles much larger than the Debye length, and applies equally well to chemical and temperature gradients [40]. This limit has been discussed previously for thermophoresis of non-ionic particles $[5,41]$ and confirmed experimentally for nanospheres in toluene [42].

The effective radius is that of the particle, $R_{\mathrm{eff}}=R_{P}$, resulting in a Soret coefficient

$$
S_{T}=\beta \frac{4 R_{P}}{3 d_{0}} \frac{H_{P S}-F_{S} H_{S S}}{k_{B} T} .
$$

The linear variation with the particle radius is related to a constant mobility [40] and consitutes one of the few rigorous results for colloidal transport.

The first term in (24) is due to solute-solvent dispersion forces that attract the particle to regions of higher density and thus to lower temperature. The second one, which describes solvent-solvent interactions, carries a minus sign. Its reduction factor $F_{S}=F\left(d_{0} / R_{S}\right)$ accounts for finite integration volume of the potential energy density: The smaller the particle volume, the weaker are the dispersion forces exerted by one solvent molecule on its neighbor. Fig. 3 shows a significant reduction for $d_{0} / R>0.2$. The above discussion suggests that the cutoff parameter $d_{0}$ is comparable to the molecular radius $R_{S}$ and that the shape factor $F_{S}$ is small. Setting $F_{S}=0$ we recover the particle mobility to the result obtained previously for a structureless solvent [19].

\section{B. Dilute polymer solutions}

At low dilution we set $\phi_{1}=0$ in (20) and thus find

$$
D_{T}=\frac{2 \beta}{9 \pi \eta d_{0}}\left(F_{P} H_{P S}-F_{S} H_{S S}\right),
$$

TABLE I: Thermophoretic mobility $D_{T}$ of different polymers in cyclooctane $\mathrm{C}_{8}$. The second row compares the mass of a monomer to that of a solvent molecule, $m=104$. The data are from Ref. [55].

\begin{tabular}{|l|c|c|c|}
\hline Polymer/solvent & $\mathrm{PE} / \mathrm{C}_{8}$ & $\mathrm{PDMS} \mathrm{C}_{8}$ & $\mathrm{PS} / \mathrm{C}_{8}$ \\
\hline$m(\mathrm{amu})$ & $28 / 104$ & $74 / 104$ & $104 / 104$ \\
\hline$D_{T}\left(\mu \mathrm{m}^{2} / \mathrm{sK}\right)$ & -2 & -1.2 & 2.7 \\
\hline
\end{tabular}

where the subscripts indicate the properties of a polymer building unit and of a solvent molecule. Like other transport coefficients, the thermophoretic mobility of high polymers is independent of the molecular weight [43-53].

The mobility may be positive or negative, depending on the form factors and Hamaker constants. Comparison with our previous work reveals a difference concerning the term proportional to $\beta H_{S S}$ : In the approach of Ref. [54] this contribution is proportional to the inverse gyration radius and thus vanishes for high polymers. The present work treats solute and solvent motion on an equal footing, and leads to the more satisfactory form (26) where the prefactor of $\beta H_{S S}$ is independent of the molecular weight.

Note that the form factor $F_{P}$ describes a single mer. For polymers made of large and heavy building blocks, such as polystyrene, one expects a positive mobility. If a single mer is smaller than a solvent molecule, one has $F_{P}<F_{S}$, and a negative mobility. In physicial terms, because of their stronger van der Waals potential, the solvent molecules diffuse more rapidly to the cold and thus push the polymer to regions of higher temperature. This argument explains, at least qualitatively, the mobilities of different polymers in cyclyoctane reported in Ref. [55]; Table 1 shows that the polyethylene (PE) and poly-dimethyl-siloxane (PDMS) are built of small molecular units and diffuse to higher temperatures $\left(D_{T}<0\right)$, whereas the heavier polystyrene has a positive mobility.

An analysis of $D_{T}$ of polystyrene in various solvents in terms of (19) with $F=1$, led to values for $d_{0}$ that range from 0.6 to $1 \mathrm{~nm}$, and correspond to the size of a solvent molecule [8]. Eq. (26) shows that the sign of the mobility is determined by the ratio $R_{P} / R_{S}$, independently of the precise value of $d_{0}$. Even if the value of $d_{0}$ and the form of the function $F$ should be taken with caution, this equation indicates the tendency that the larger molecular units diffuse to lower $T$. There are additional effects arising from the different Hamaker constants, weak hydrodynamic interactions between neighor beads, and the kinetic energy.

The mutual diffusion coefficient at low dilution reads $D=k_{B} T / 6 \pi \eta R_{h}$, where $R_{h}$ is the hydrodynamic radius of the polymer chain. With respect to the composition and the molecular radii, the resulting Soret coefficient

$$
S_{T}=\beta \frac{4 R_{h}}{3 d_{0}} \frac{F_{P} H_{P S}-F_{S} H_{S S}}{k_{B} T}
$$

behaves like the mobility $D_{T}$. It is proportional to the 
hydrodynamic radius of the polymer coil and thus increases with the chain length; this dependence has been observed for polystryene in different solvents [44].

\section{Molecular mixtures}

We briefly discuss the three factors appearing in (22) for organic molecules of comparable size. For most liquids, the thermal expansion coefficient $\beta$ takes values of about $10^{-3} \mathrm{~K}^{-1}$. According to the above discussion of the cut-off parameter, the ratio $R_{\text {eff }} / d_{0}$ is not very different from unity. Finally, the last factor expresses the effective Hamaker constant in units of the thermal energy; with typical values for $H_{i} \sim 5 k_{B} T$ and $F_{i}$ from Fig. 2, one finds numbers of the order of 1 but hardly larger than 4.

As noted in previous works, $S_{T}$ is proportional to the thermal expansivity [5, 41, 56-58]; the remaining factors in (22) depend on molecular details and in particular on the composition. Indeed, the Soret coefficient of mixtures of aliphatic and aromatic molecules is mostly hardly exceeds a few $10^{-3} \mathrm{~K}^{-1}$.

Fig. 4 shows the Soret coefficient as a function of the composition $\phi$ for three values of the molecular size ratio. In all cases the cut-off parameter $d_{0}$ is equal to the smaller radius. If both species are of equal size, the effective radius $R_{\text {eff }}$ is constant, and the Hamaker constants result in a linear variation with $\phi$. Unlike molecular radii result in a non-uniform slope, as illustrated by the upper and lower curves. A rather similar behavior has been observed for binary liquids $[13,15]$. For molecules of equal size, the dependence on the interaction parameters agrees well with numerical simulations [29].

In order to separate the dependencies of (22) on composition, size, and Hamaker constants, we consider in the following several special cases.

\section{Composition dependence at equal size}

If both molecular species have the same size, the form factors are identical, $F=F_{1,2}$, and simplifiy the mobility

$$
S_{T}=\beta \frac{4 R}{3 d_{0}} F \frac{H_{0}+\phi_{1} \delta H}{k_{B} T},
$$

where we have used $\phi_{2}=1-\phi_{1}$, and the shorthand notation $H_{0}=H_{12}-H_{22}$ and $\delta H=H_{11}+H_{22}-2 H_{12}$. As a remarkable property, this mobility varies linearly with the volume fraction $\phi_{1}$. The parameters $H_{0}$ and $\delta H$ may be positive or negative, and may result in a change of sign of $S_{T}$ as a function of $\phi_{1}$. This is illustrated by the middle curve of Fig. 4.

A roughly linear dependence on $\phi_{1}$ has been reported for mixtures of benzene, toluene, and cyclohexane [13]; more complexe variations occur for flexible alkane chains, sugars, and polar molecules such as acetone [14-18].

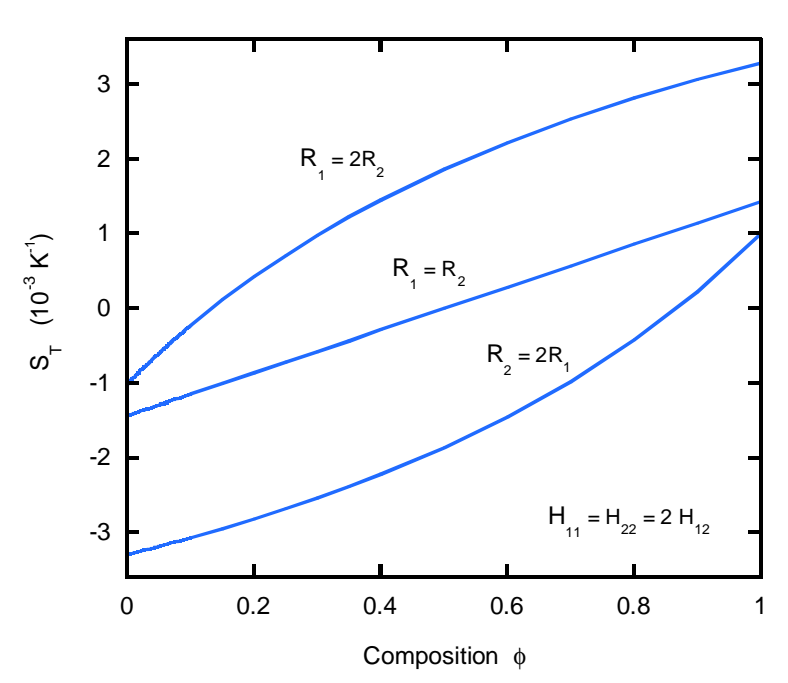

FIG. 4: Soret coeffiicient as a function of the composition. The radii are equal to the cutoff parameter $d_{0}$, or twice $d_{0}$; the off-diagonal Hamaker constant is $H_{12}=3 k_{B} T$, and the thermal expansivity $\beta=10^{-3} \mathrm{~K}^{-1}$.

Both the linear variation with composition and the dependence on the Hamaker constants agree with numerical simulations of a Lennard-Jones binary liquid [32].

\section{E. Size dependence at equal Hamaker constants}

If the components have similar chemical properties, their Hamaker constants do not vary much. When setting $H=H_{i j}$ for all pair interactions, the mobility takes a particularly simple form

$$
S_{T}=\beta \frac{4\left(F_{1}-F_{2}\right)}{3 d_{0}\left(\phi_{1} / R_{2}+\phi_{2} / R_{1}\right)} \frac{H}{k_{B} T},
$$

that is proportional to the difference of the form factors of the two species. Since $F$ is a mononotically increasing function of the molecular radius, this means that the smaller species migrates to the warm, and the larger one to the cold. The second factor depends on the composition; the Soret coefficient is larger if the smaller species is more abundant.

Real molecules are not spherical beads, and their size cannot be reduced to a single radius. Still, the radius dependence of the form factor expresses the dependence on the molecular volume. Since most organic molecules have rather similar densities, $R$ may be equally well related to the molecular mass; in the simplest case one has $m \propto R^{3}$. Then (29) states that the heavier species migrates to the cold, and the lighter one to the warm. This rule is confirmed by Soret data on various binary mixtures [14].

In their numerical simulation results of Lennard-Jones liquids, Galliéro et al. found that the larger species mi- 
grate to the warm [29], which at first sight seems to contradict the present result (29). Yet in view of the different interaction potentials, this discrepancy is not surprising: The simulations of Ref. [29] are based on a single atomatom Lennard-Jones potential $\varphi_{L J}=\varepsilon\left[(\sigma / r)^{12}-(\sigma / r)^{6}\right]$; the size dependence is obtained by varying the length $\sigma$ at constant interaction parameter $\varepsilon$. The present work describes the molecular interactions in terms of the van der Waals potential (10) which reduces to the LennardJones form $\sim 1 / r^{6}$ in the limit $r \gg R$, but shows a more complex variation at short distances. The Lennard-Jones potential results in a force $-\nabla \varphi_{L J} \sim \varepsilon / \sigma$ that becomes weaker as the parameter $\sigma$ increases. On the other hand, the force derived from (10) increases with the molecular radius.

\section{F. Kinetic energy}

In this paper we have only considered dispersion forces and neglected the thermal agitation of the molecules. That is why the Soret coefficient disappears in the absence of van der Waals interactions. On the contrary, thermal diffusion in gas mixtures and aerosols is well described by kinetic theory. Much numerical work has been done on hard-sphere systems [30, 59].

From recent work on a simple hard-bead model it is clear that the kinetic energy contributes significantly to the Soret coefficient in liquids, and may even dominate in the case of molecular mixtures [22]. The isotope effect observed upon deuteration or replacing common carbon ${ }^{12} \mathrm{C}$ with the heavier isotope ${ }^{13} \mathrm{C}$ benzene, indicates that the Soret coefficient consists of a "chemical" contribution and a term depending on the molecular masses [13, 32].

Taking their sum one finds the total Soret coefficient

$$
S_{T}^{\mathrm{tot}}=S_{T}+S_{T}^{\mathrm{mass}}
$$

where the chemical contribution $S_{T}$ is obtained in the present work and the mass-dependent one has been derived in [22]. For small molecules, both may be of comparable size. Thus a quantitative fit of experimental data should include both contributions. Yet so far the kinetic contribution has been derived only for binary mixtures where both components are of equal size [22]; one may expect that a size difference will signficantly affect the mass dependent term.

\section{SUMMARY AND CONCLUSION}

Starting from the well-known expression for large solute particles at low dilution, we have studied the dependence of the Soret coefficient on the composition and on the molecular size. As a main result, the form factors $F$ account for the fact that smaller beads have a weaker van der Waals potential, and reduce the Soret motion accordingly. As a general law, we find that the smaller species migrates to the warm, due to its weaker dispersion forces; this mechanism is fundamentally different from the mass effect discussed in $[22,28,29]$. In the limit of large particles, we find that the mobility $D_{T}$ becomes independent of size, in accord with colloidal transport theory [40].

A second important aspect concerns the variation of $S_{T}$ with composition. Our findings show a rather complex behavior that arises from the mutual diffusion coefficient (8) and the effective Hamaker parameters, as illustrated in Fig. 4. The change of sign is due to the composition dependence of the Hamaker coefficients. Most of our results agree with previous numerical simulation studies on Lennard-Jones binary liquids [28, 29, 32].
[1] S.R. de Groot, P. Mazur, Non-equlibrium Thermodynamics, North Holland Publishing, Amsterdam (1962)

[2] W. Köhler, S. Wiegand, Thermal Nonequilibrium Phenomena in Fluid Mixtures, Lecture Notes in Physics Vol. 584, Springer, Berlin (2002)

[3] S. Wiegand, J. Phys. Cond. Matt. 16, 357 (2004)

[4] J.K. Platten, J. Appl. Mech. 73, 5 (2006)

[5] H. Brenner, Phys. Rev. E 74, 036306 (2006)

[6] R. Piazza, A. Parola, J. Phys.: Condens. Matter 20, $153102(2008)$

[7] R. Piazza, Soft Matter 4, 1740 (2008)

[8] A. Würger, Rep. Prog. Phys. 73, 126602 (2010)

[9] E. Ruckenstein, J. Colloid Interface Sci. 83, 77 (1981)

[10] S.A. Putnam, D.G. Cahill, Langmuir 21, 5317 (2005)

[11] A. Würger, Phys. Rev. Lett. 101, 108302 (2008)

[12] D. Vigolo, S. Buzzaccaro, R. Piazza, Langmuir (2010)

[13] C. Debuschewitz, W. Köhler, Phys. Rev. Lett. 87, 055901 (2001)

[14] G. Wittko, W. Köhler, J. Chem. Phys. 123, 014506
$(2005)$

[15] G. Wittko, W. Köhler, Europhys. Lett. 78, 46007 (2007)

[16] P. Polyakov, E. Rossinsky, S. Wiegand, J. Phys. Chem. B 113, 13308 (2009)

[17] P. Blanco, P. Polyakov, M. Mounir Bou-Ali, S. Wiegand, J. Phys. Chem. B 112, 8340 (2010)

[18] P. Blanco, H. Kriegs, B. Arlt, S. Wiegand, J. Phys. Chem. B 114, 10740 (2010)

[19] A. Würger, Langmuir 25, 6696 (2009)

[20] S.N. Semenov, M.E. Schimpf, Phys. Rev. E 69, 011201 (2004)

[21] K.I. Morozov, Phys. Rev. E 79, 031204 (2009)

[22] S. Villain-Guillot, A. Würger, Phys. Rev. E 83, 030501 (2011)

[23] E.L. Dougherty, H.G. Drickamer, J. Phys. Chem. 59, 443 (1955)

[24] K. Shukla, A. Firoozabadi, Ind. Chem. Eng. Res. 37, 3331 (1998)

[25] L.J.T.M. Kempers, J. Chem. Phys. 115, 6330 (2001) 
[26] M. Eslamian, M.Z. Saghir, Phys. Rev. E 80, 0661201 (2009)

[27] B. Hafskjold, T. Ikeshoji, S.K. Ratkje, Mol. Phys. 80, 1389 (1993)

[28] F. Müller-Plathe, D. Reith, Comp. Theor. Polym. Sci. 9, 203 (1999)

[29] G. Galliero et al., Fluid Phase Equ. 208, 171 (2003)

[30] S. Yeganegi, M. Zolfaghari, Fluid Phase Equilib. 243, $161(2006)$

[31] M. Zhang, F. Müller-Plathe, J. Chem. Phys. 125, 124903 (2006)

[32] P.-A. Artola, B. Rousseau, Phys. Rev. Lett. 98, 125901 (2007)

[33] P.-A.. Artola, B. Rousseau, G. Galliéro, J. Am. Chem. Soc. 130, 10963 (2008)

[34] G. Galliero, S. Volz, J. Chem. Phys 128, 064505 (2008)

[35] R.J. Bearman, J.G. Kirkwood, J. Chem. Phys. 28, 136 (1958)

[36] E. Helfand, J.G. Kirkwood, J. Chem. Phys. 32, 857 (1960)

[37] G.S. Hartley, K. Crank, Trans. Faraday Soc. 52, 781 (1956).

[38] A.L. van Geet, A.W. Adamson, J. Phys. Chem. 68, 238 (1964).

[39] W.B. Russel, D.A. Saville, W.R. Schowalter, Colloidal Dispersions, Cambridge University Press (1989)

[40] J. L. Anderson, Ann. Rev. Fluid Mech. 21, 61 (1989)

[41] J.R. Bielenberg, H. Brenner, Physica A 356, 279 (2005)
[42] R. Spill, W. Köhler, G. Lindenblatt, W. Schartl, Phys. Rev. E 62, 8361 (2000)

[43] N.T. Khazanovich, J. Polym. Sci. Part C 16, 2463 (1967)

[44] J.C. Giddings et al., Macromolecules 9, 106 (1976)

[45] F. Brochard, P.-G. de Gennes, C. R. Acad. Sc. Paris, Série II 293, 1025 (1981)

[46] M.E. Schimpf, J.C. Giddings, Macromolecules 20, 1561 (1987)

[47] P. Rossmanith, W. Köhler, Macromolecules 29, 3203 (1996)

[48] J. Rauch, W. Köhler, Phys. Rev. Lett. 88, 185901 (2002)

[49] J. Chan et al., J. Solution Chem. 32, 197 (2003)

[50] J. Rauch, W. Köhler, Macromolecules 38, 3571 (2005)

[51] M. Hartung, J. Rauch, W. Köhler, J. Chem. Phys. 125, 214904 (2006).

[52] A. Würger, Phys. Rev. Lett. 98, 138301 (2007)

[53] D. Stadelmaier, W. Köhler, Macromolecules 41, 6205 (2008)

[54] A. Würger, Phys. Rev. Lett. 102, 078302 (2009).

[55] D. Stadelmaier, W. Köhler, Macromolecules 42, 9147 (2009)

[56] R. Piazza, J. Phys.: Condens. Matter 16, 4195 (2004)

[57] S.N. Semenov, M.E. Schimpf, Phys. Usp. 52, 1045 (2009)

[58] H. Brenner, Phys. Rev. E 82, 036325 (2010)

[59] J.M. Kincaid, E.G.D. Cohen, M. Lopez de Haro, J. Chem. Phys 86, 963 (1987) 